DOI: 10.20472/IAC.2018.041.012

\author{
ANUP DEWANJI \\ Indian Statistical Institute , India \\ KIT CURTIUS \\ Centre for Tumour Biology, Barts Cancer Institute, United Kingdom \\ WILLIAM H HAZELTON \\ Fred Hutchinson Cancer Research Center, United States \\ GEORG LUEBECK \\ Fred Hutchinson Cancer Research Center, United States

\section{OPTIMAL ADAPTIVE SCREEN DESIGN FOR BARRETT'S ESOPHAGUS (BE)}

\begin{abstract}
:
The incidence rate of Esophageal Adenocarcinoma (EAC), which occurs at the distal end of the esophagus near the junction with the stomach, has increased 5-6 fold over the past four decades. EAC arises primarily in Barrett's esophagus (BE), a metaplastic tissue alteration in the esophageal lining, frequently associated with chronic symptoms of gastroesophageal reflux disease (GERD), which exposes the distal esophagus to bile salts and stomach acid. BE progresses through low grade and then high grade dysplasia, and finally forms small malignant cell populations that progresses to invasive cancer. Majority of BE patients remain undiagnosed and thus most EAC cases are diagnosed at an advanced stage. This is an unfortunate reality because mortality associated with EAC is very high. The main idea of screening is early detection so that there is an opportunity to change its prognosis, which is greatly improved for BE patients with high grade dysplasia (HGD) and/or cancer that is detected at an early stage. However, on average, only $0.2-0.5 \%$ of people with BE develop EAC. Thus, the majority of BE patients who undergo regular screening will not develop EAC in their lifetimes, indicative of over-screening. Therefore, there is a need for finding an optimal screening strategy.
\end{abstract}

We suggest an adaptive screen design for Barrett's Esophagus (BE) based on the multi-stage clonal expansion (MSCE) model. Certain 'windows of opportunity' exist during progression from Barrett's Esophagus (BE) to esophageal adenocarcinoma (EAC) in which curative interventions can be performed. Analytical and flexible formulae for the probability of being in a certain screening window allows straightforward maximization for determining optimal screening time and subsequent decision-making. Adaptive screening design incorporates patient-specific details to optimize the time until next screen. Several examples will be considered for illustration.

\title{
Keywords:
}

Barrett's Esophagus, Esophageal Adenocarcinoma, Multi-stage Clonal Expansion Model, Adaptive Screening Design. 